ISSN 0103-5150

Fisioter. Mov., Curitiba, v. 26, n. 1, p. 167-174, jan./mar. 2013 Licenciado sob uma Licença Creative Commons

\title{
Aplicação de exercício isotônico durante a hemodiálise melhora a eficiência dialítica
}

\author{
Isotonic exercise during hemodialysis improves \\ dialysis efficiency
}

\begin{abstract}
Ana Paula Coelho Figueira Freire ${ }^{[a]}$, Cibele Silva Rios ${ }^{[b]}$, Raquel Souza Moura ${ }^{[b]}$, Regina Coeli Vasques de Miranda Burneiko ${ }^{[c]}$, Susimary Aparecida Trevizan Padulla ${ }^{[\mathrm{d}]}$, Francis da Silva Lopes ${ }^{[\mathrm{e}]}$
\end{abstract}

[a] Mestranda em Fisioterapia pela Universidade Estadual Paulista (Unesp), Presidente Prudente, SP - Brasil, e-mail: anapcff@hotmail.com

[b] Especialistas em Fisioterapia Cardiorrespiratória pela Universidade do Oeste Paulista (Unoeste), Presidente Prudente, SP - Brasil, e-mail: cibelerios@hotmail.com, e-mail: raquelsmoura@gmail.com

[c] Doutora em Fisiopatologia em Clínica Médica pela Universidade Estadual Paulista (Unesp), docente do Departamento de Fisioterapia da Universidade Estadual Paulista (Unesp), Presidente Prudente, SP - Brasil, e-mail: susi@fct.unesp.br

[d] Doutora em Ciências da Saúde pela Faculdade de Medicina de São José do Rio Preto (Famerp), docente do Departamento de Fisioterapia da Universidade Estadual Paulista (Unesp), Presidente Prudente, SP - Brasil, e-mail: re.miranda@fct.unesp.br

[e] Doutora em Biologia Celular e Estrutural pela Universidade Estadual de Campinas (Unicamp), professora do Departamento de Fisioterapia da Universidade do Oeste Paulista (Unoeste), Presidente Prudente, SP - Brasil, e-mail: francispacagnelli@unoeste.br

\section{Resumo}

Introdução: A doença renal crônica (DRC) é decorrente de uma lesão renal e perda progressiva e irreversível da função dos rins. A hemodiálise substitui parcialmente essa função, com o objetivo de corrigir as alterações metabólicas na DRC. Para acompanhar a adequação da diálise, é determinado o Kt/V - índice de depuração da ureia por sessão de hemodiálise. 0 exercício físico de moderada intensidade tem se mostrado de fundamental importância para melhorar os efeitos adversos ao tratamento dialítico. Objetivos: Avaliar o Kt/V em indivíduos com DRC submetidos ao exercício físico isotônico de baixa intensidade durante a hemodiálise. Materiais e métodos: Analisados dados de 15 voluntários de ambos os sexos, submetidos à hemodiálise três vezes por semana. Após duas horas do início da diálise, foi aplicado um protocolo de exercícios 
isotônicos de baixa intensidade de membros superiores e inferiores com duração de 30 minutos, por um período de três meses. Os valores do Kt/V foram comparados no período de três meses anteriores sem exercício e após três meses de exercício. Resultados: A média dos valores do Kt/V nos três meses sem exercício foi de 1,13 \pm 0,11 e após aplicação do programa de exercícios foi de 1,29 $\pm 0,12(p<0,05)$. Conclusão: O programa de exercício físico isotônico de baixa intensidade em pacientes com DRC, aplicados durante a sessão de diálise mostrou a melhora da eficiência dialítica.

Palavra-chaves: Doença renal crônica. Hemodiálise. Exercício físico.

\section{Abstract}

Introduction: The chronic kidney disease (CKD) is due to a renal injury and progressive and irreversible loss of kidney function. Hemodialysis replace partially this function with the objective of correcting the metabolic changes in the DRC. To monitor the adequacy of dialysis is determined the Kt/V-rate of clearance of urea per session of dialysis. The exercise of moderate intensity has been of fundamental importance for improving the adverse effects of dialysis treatment. Objectives: To evaluate Kt/V in persons with CKD submitted the isotonic exercise of low intensity during hemodialysis. Materials and methods: It was analyzed data from 15 volunteers with CKD, both sexes, submitted to dialysis three times a week. After two hours of the start of dialysis, was applied a protocol of isotonic exercises with low intensity of upper and lower limbs lasting 30 minutes, for a period of three months. The values of Kt/V were compared in the period of three months without exercise and after three months (monthly average). The paired Student $t$ test was used for statistical analysis $(p<0.05)$. Results: The average values of Kt $/ V$ within three months without exercise was $1.13 \pm 0.11$ and after implementation of the program of exercises was $1.29 \pm 0.12(p<0.05)$. Conclusion: The program of isotonic exercises of low intensity in patients with CKD applied during the session of dialysis improved the efficiency dialysis.

Keywords: Chronic Kidney disease. Hemodialysis. Physical activity.

\section{Introdução}

A doença renal crônica (DRC) é caracterizada como uma síndrome clínica causada pela perda progressiva e irreversível das funções renais (glomerular, tubular e endócrina). Suas primeiras fases podem ser detectadas em exames laboratoriais de rotina $(1,2,3,4)$.

A DRC tornou-se um grande problema de saúde pública no mundo todo; cerca de 1,2 milhões de pessoas encontram-se sob tratamento dialítico. No Brasil, são aproximadamente 54,5 mil pessoas (5). No ano de 2002, segundo dados da Sociedade Brasileira de Nefrologia (4), o custo anual com esses pacientes foi de R $\$ 1,1$ bilhão. Estimativas de diferentes países indicam que mais de $60 \%$ dos pacientes morrem antes de completar 10 anos de diálise $(6,7)$.

A hemodiálise substitui parcialmente a função dos rins, com o objetivo de corrigir as alterações metabólicas observadas na DRC, a partir da filtração sanguínea, removendo, com isso, os solutos urêmicos através de um gradiente de concentração por difusão ou ultrafiltração, restabelecendo o equilíbrio hidroeletrolítico e ácido-básico do organismo. Para verificar a eficiência da hemodiálise realizada e acompanhar a sua adequação, é determinado o Kt/V, ou índice de depuração da ureia por sessão de hemodiálise (8).

O controle ideal do paciente portador de DRC em diálise crônica é bastante complexo. Apesar das deficiências da aplicabilidade do Kt/V em pacientes com DRC, o índice continua sendo usado devido à inexistência de um melhor. Em suma, o Kt/V expressa quantas vezes o volume de distribuição da ureia foi depurado; o resultado é obtido pela multiplicação da depuração do dialisador (clearance; $\mathrm{K}$, em mL/min) pelo tempo de diálise previsto ( $\mathrm{t}$ em min), e divide-se o resultado pelo volume de distribuição de soluto do paciente (V em $\mathrm{mL}$; para a ureia este corresponde a $60 \%$ do peso do paciente). Utilizam-se fórmulas indiretas; a mais aceita entre elas é a proposta por Daugirdas, em que Kt $/ \mathrm{V}=\mathrm{Ln}(\mathrm{R}-0.008 \times \mathrm{t})+(4-3.5 \times$ $\mathrm{R}) \times \mathrm{UF} / \mathrm{P}$, onde $\mathrm{Ln}=$ logaritmo natural, $\mathrm{t}=$ duração em horas da sessão, $\mathrm{R}=$ relação entre ureia sérica pós e pré-diálise em $\mathrm{mg} / \mathrm{dL}, \mathrm{UF}=$ volume ultrafiltrado em litros, e $\mathrm{P}=$ peso pós-diálise em kg. Para os portadores 
de DRC mantidos em programa de hemodiálise, os valores do Kt/V maiores que 1,2 estão relacionados a uma morbidade e mortalidade reduzida $(2,3,8,9)$.

Um dos fatores que podem contribuir para alterar a eficiência dialílitca é o efeito rebote. Porque a hemodiálise é realizada com o indivíduo em repouso, posição em que a circulação está relativamente estagnada, é gerado um atraso no equilíbrio de ureia durante a diálise, limitando sua eficácia $(10,11)$. Durante a diálise, a ureia é removida rapidamente do sangue, mas se mantém de forma desproporcional nos compartimentos da periferia do corpo, principalmente nos membros inferiores $(12,13)$. Após a hemodiálise a concentração plasmática de ureia aumenta de forma rápida, levando a um reequilíbrio da ureia intracelular, e essa retenção se deve à sua baixa difusão ao redor da membrana celular ou ao baixo fluxo sanguíneo em alguns compartimentos do corpo $(14,15,16)$.

0 exercício físico é uma das propostas para, durante a hemodiálise, ativar a circulação que se encontra estagnada e, consequentemente, diminuir o efeito rebote e melhorar a eficiência dialítica. Realizando exercícios com movimentos corporais como alongamentos, calistênicos e isotônicos, ocorre um aumento da oxigenação, temperatura e contração muscular, ocorrendo a dilatação dos capilares que estavam constritos, aumentando a circulação $(17,18,19,20)$.

Além de efeitos vasodilatadores, a atividade física tem sido proposta para pacientes com DRC $(21,22$, 23) para melhorar o condicionamento físico $(24,25$, $26,27)$, a capacidade funcional $(23,27,28)$, a força muscular $(26,27,28)$ e a qualidade de vida desses pacientes $(29,30)$. Dessa forma, a atividade física para nefropatas crônicos tem sido introduzida nas diretrizes de reabilitação, com grau de recomendação B (deve ser geralmente indicada) e nível de evidência 3 (baseada em poucos estudos randomizados) (31).

Portanto, o objetivo deste estudo foi avaliar os efeitos da atividade física de baixa intensidade durante a hemodiálise nas alterações do Kt/V.

\section{Materiais e métodos}

População e amostra

Este foi um estudo retrospectivo analisado a partir dos prontuários dos pacientes. Todos os envolvidos neste projeto participaram de um tratamento fisioterapêutico que foi constituído de exercício físico isotônico de baixa intensidade durante a hemodiálise e assinaram um Termo de Consentimento Livre e Esclarecido. Esta pesquisa foi aprovada pelo Comitê de Ética Institucional (protocolo n. 113/07) e obedece à Resolução n. 196/96.

Foram analisados dados de pacientes de ambos os sexos, portadores de DRC, submetidos à hemodiálise clássica três vezes por semana, quatro horas por sessão, no Instituto do Rim de Presidente Prudente (SP).

Os critérios de exclusão foram descompensações hemodinâmicas, mudanças no acesso à hemodiálise, no tempo de hemodiálise, no volume ultrafiltrado e no tipo de filtro utilizado.

As sessões de hemodiálise tinham alguns parâmetros fixos que foram: fluxo de sangue de $300 \mathrm{~mL} / \mathrm{min}$, fluxo de diálise de $500 \mathrm{~mL} / \mathrm{min}$, heparina aplicada em bolos $(1,25 \mathrm{~mL})$ no inicio da diálise e a cada hora de tratamento dialítico.

Para o cálculo do Kt/V, foram necessários os valores da duração da hemodiálise em horas (fixo em 4 horas), o valor da ureia sérica pré e pós-hemodiálise (dosada por exame de sangue), peso do paciente pós-hemodiálise e volume ultrafiltrado (diferença do peso pré e pós-hemodiálise). Esse parâmetro é avaliado mensalmente.

Os exercícios propostos tiveram duração de 30 minutos, por um período de três meses. Os valores do Kt/V foram comparados no período de três meses anteriores, obtidos de uma sessão em cada mês sem exercício (média) e durante os três meses de exercício obtidos de uma sessão a cada mês (média).

\section{Avaliação inicial}

A avaliação fisioterapêutica abordou idade, escolaridade, comorbidades, tempo em que realiza hemodiálise, se já foi submetido a um transplante renal e etiologia da doença renal crônica (Quadro 1).

O exame físico consistiu em inspeção, palpação, avaliação de amplitude de movimento articular e de dados vitais como frequência cardíaca, frequência respiratória, determinação da pressão arterial e ausculta cardíaca e pulmonar.

\section{Protocolo experimental}

Antes, durante e após as terapias, os dados vitais foram monitorados. Um protocolo de exercício 
físico de componente isotônico de baixa intensidade foi elaborado para membros superiores e inferiores (18): Esse protocolo constou de autoalongamento, exercícios isotônicos e relaxamento com musicoterapia. Foram alongados os músculos cervicais, flexores e extensores do punho, executados duas vezes por trinta segundos durante a hemodiálise. Os exercícios isotônicos para membros superiores foram realizados de modo alternado - flexão e extensão de cotovelo e flexão de dedos, pressionando uma bola. Para membros inferiores, foram realizadas tríplice flexão, flexão, extensão, adução e abdução de quadril, flexão e extensão de joelho, dorsiflexão e flexão plantar de tornozelo. A última fase foi a de relaxamento, que consistiu na circundação do pescoço, rotação interna e externa do ombro; respirações lentas e profundas e técnicas lúdicas.

Todas as sessões foram realizadas durante a hemodiálise, com duração média de 30 minutos com musicoterapia, promovendo mudança na rotina dos pacientes e descontração. As sessões foram conduzidas por um fisioterapeuta, com a participação de alunos da graduação em Fisioterapia.

Quadro 1 - Caracterização dos doentes renais crônicos submetidos a um protocolo de exercícios isotônicos durante a hemodiálise. Frequência absoluta e percentual

(Continua)

\begin{tabular}{lcc}
\hline \multicolumn{3}{c}{ Doentes Renais Crônicos $(\mathrm{n}=\mathbf{1 5})$} \\
\hline Idade (anos) & $\mathbf{n}$ & $\%$ \\
\hline $20-30$ & 01 & 6,66 \\
$41-50$ & 02 & 13,33 \\
$51-60$ & 04 & 26,66 \\
$61-70$ & 05 & 33,33 \\
Acima de 71 & 03 & 20,00 \\
\hline Escolaridade & & \\
\hline Analfabeto & 06 & 40,00 \\
Ensino Fundamental I & 07 & 46,66 \\
Ensino Fundamental C & 01 & 6,66 \\
Ensino Médio C & 01 & 6,66 \\
\hline Comorbidades & & \\
\hline Hipertensão & 11 & 73,00 \\
Diabetes Mellitus & 06 & 40,00 \\
\hline
\end{tabular}

\section{Análise estatística}

A análise estatística utilizada foi efetuada a partir do teste $t$-Student pareado, $(\mathrm{p}<0,05)$, considerando que os mesmos pacientes apresentaram resultados antes e após o período de fisioterapia. Os dados foram expressos em média e desvio padrão.

\section{Resultados}

Foram considerados os parâmetros médios dos pacientes em períodos de tempo iguais: três meses sem fisioterapia, e três meses com a fisioterapia, para o Kt/V, peso pré e pós-diálise, volume ultrafiltrado, ureia pré e pós-hemodiálise.

0 peso do paciente no mês sem exercícios antes da hemodiálise foi de $69,69 \pm 14,68$ kg e após a hemodiálise foi $68,44 \pm 14,55 \mathrm{~kg}$. 0 peso dos pacientes no mês com exercícios antes da hemodiálise foi de 69,92 \pm $14,55 \mathrm{~kg}$ e após a hemodiálise foi $68,49 \pm 14,46 \mathrm{~kg}$. Não houve diferença estatisticamente significativa (Quadro 2).

Quadro 1 - Caracterização dos doentes renais crônicos submetidos a um protocolo de exercícios isotônicos durante a hemodiálise. Frequência absoluta e percentual

(Conclusão)

\begin{tabular}{lcc}
\hline \multicolumn{3}{c}{ Doentes Renais Crônicos $(\mathrm{n}=15)$} \\
\hline $\begin{array}{l}\text { Tempo em hemodiálise } \\
\text { (anos) }\end{array}$ & $\mathrm{N}$ & $\%$ \\
\hline $2-4$ & 07 & 46,60 \\
$5-6$ & 05 & 33,33 \\
$7-8$ & 03 & 19,98 \\
\hline $\begin{array}{l}\text { Transplante renal } \\
\text { (há 4 anos) }\end{array}$ & 01 & 6,66 \\
\hline Etilogia da doença renal & & \\
\hline Glomerulonefrite Crônica & 04 & 26,66 \\
Nefropatia Diabética & 01 & 6,66 \\
Nefroesclerose Hipertensiva & 07 & 46,66 \\
Rins Policísticos & 01 & 6,66 \\
Pielonefrite Crônica & 01 & 6,66 \\
\hline
\end{tabular}

Fonte: Dados da pesquisa. 
Quadro 2 - Parâmetros analisados (média \pm desvio padrão) nos 3 meses com e sem exercício isotônico

\begin{tabular}{lcc}
\hline Determinantes & Sem exercício & Com exercício isotônico \\
\hline Peso pré-diálise $(\mathrm{kg})$ & $69,69 \pm 14,68$ & $69,92 \pm 14,55$ \\
Peso pós-diálise $(\mathrm{kg})$ & $68,44 \pm 14,55$ & $68,49 \pm 14,46$ \\
Volume ultrafiltrado $(\mathrm{l})$ & $1,25 \pm 0,74$ & $1,13 \pm 0,70$ \\
Ureia pré-diálise $(\mathrm{mg} / \mathrm{dL})$ & $112,80 \pm 34,16$ & $124,02 \pm 29,03$ \\
Ureia pós-diálise $(\mathrm{mg} / \mathrm{dL})$ & $41,04 \pm 16,15^{\star}$ & $43,53 \pm 13,61^{*}$ \\
Kt/V & $1,13 \pm 0,11$ & $1,29 \pm 0,12^{\star *}$ \\
\hline
\end{tabular}

Legenda: * $=$ Comparado ureia pré com pós-hemodiálise $\mathrm{p}<0,05 ;{ }^{* *}=$ Comparado Kt $\mathrm{V}$ sem e com exercícios.

Fonte: Dados da pesquisa.

0 volume ultrafiltrado no mês sem exercício foi $1,25 \pm 0,74$ litros comparado com o mês com exercício, que foi de 1,13 $\pm 0,70$ litros. Não foi estatisticamente significativo.

A avaliação da ureia sérica foi realizada antes e após a hemodiálise para o posterior cálculo do $\mathrm{Kt} / \mathrm{V}$. A quantidade média de ureia nos meses sem exercício pré-hemodiálise foi de $112,80 \pm 34,16 \mathrm{mg} /$ $\mathrm{dL}$ e após a hemodiálise foi de 41,04 $\pm 16,15 \mathrm{mg} / \mathrm{dL}$ $(\mathrm{p}<0,05)$. Nos meses com exercício, o valor da ureia pré-hemodiálise foi de $124,02 \pm 29 \mathrm{mg} / \mathrm{dL}$ e após a hemodiálise foi de 43,53 $\pm 13,61 \mathrm{mg} / \mathrm{dL}$. Não houve diferença estatisticamente significativa comparando os valores da ureia pré-hemodiálise nos meses com e sem exercício isotônico.

Os valores médios do índice de depuração da ureia, Kt/V, nos meses sem exercício foi de 1,13 \pm 0,11 . Após a aplicação do programa de exercícios, o valor do Kt/V obtido foi de 1,29 $\pm 0,12$. Houve diferença estatisticamente significativa comparando os valores do $\mathrm{Kt} / \mathrm{V}$ nos meses sem exercício com os meses com exercícios isotônicos.

\section{Discussão}

O principal achado deste estudo foi a melhora do índice de depuração da ureia (Kt/V) após a aplicação de exercícios isotônicos de baixa intensidade aplicados durante a sessão hemodialítica.

Propostas de exercícios durante a hemodiálise e seus efeitos benéficos em vários parâmetros, como por exemplo, na capacidade funcional, qualidade de vida, função muscular, desempenho físico e eficiência dialítica tem sido demonstrados principalmente em exercícios de moderada intensidade $(22,23,24,31$, $32,33,34)$.

No presente estudo, os resultados obtidos corroboram com Peres (5), que avaliou entre outros parâmetros a eficiência dialítica. Nesse estudo com 58 pacientes portadores de DRC, demonstrou-se que exercícios de baixa intensidade semelhantes ao do presente estudo, feitos três vezes por semana, durante oito semanas, com uma hora de duração, melhorou a capacidade funcional, a força muscular e a eficiência da diálise.

Parsons et al. (34) também analisaram a eficiência dialítica. Os participantes desse estudo exercitaram-se três vezes por semana, durante 60 minutos de exercício de baixa intensidade, durante 20 semanas através do ciclo ergômetro, o que difere do protocolo utilizado no presente estudo.

Outras pesquisas realizadas com cicloergômetro são descritas na literatura $(9,13)$, porém, diferente deste estudo, optou-se por não utilizar equipamentos para não onerar a proposta e torná-la viável. Nesta pesquisa, o programa de exercícios físicos isotônico de baixa intensidade durante a hemodiálise mostrou-se de fácil aplicação, sem custos e com resultados satisfatórios para potencializar o efeito do tratamento hemodialítico.

Essa melhora do Kt/V, identificada no presente estudo, pode ser atribuída ao aumento do fluxo sanguíneo durante o exercício, aumentando a perfusão muscular e a remoção de fluidos $(5,35)$. Foi demonstrado que exercício durante a diálise aumenta em $20 \%$ a remoção de ureia; isso diminuiu o efeito rebote, em razão do aumento do fluxo sanguíneo pelos tecidos durante os exercícios utilizando os membros inferiores, permitindo vasodilatação capilar e 
provendo, assim, uma maior superfície para troca de substâncias dos tecidos para o sangue $(30,34$, 35). Dessa forma, a atividade física durante a diálise provavelmente causou uma vasodilatação periférica adequada, aumentando a perfusão muscular e a área de superfície para troca de substâncias dos tecidos para o sangue, melhorando a qualidade da diálise analisada através do Kt/V (36, 37, 38, 39, 40, 41).

Uma limitação da pesquisa foi o número de pacientes envolvidos, pois durante o estudo houve a necessidade de excluir alguns dos pacientes em virtude de mudança de filtro de diálise ou intercorrências nesse período. Sugere-se que novos estudos sejam realizados para abordar novos protocolos de tratamento e, consequentemente, beneficiar os pacientes submetidos à hemodiálise.

\section{Conclusão}

Os resultados desta pesquisa sugerem que programas de exercícios de alongamentos e isotônicos de baixa intensidade em pacientes com DRC aplicados durante a sessão de diálise melhoram a eficiência dialítica. Como o aumento do índice de depuração da ureia (Kt/V) demonstra estar relacionado com menor morbidade, essa é uma proposta simples, sem custo e de fácil aplicação que pode contribuir também para melhorar a condição física desses pacientes, e sua qualidade de vida. Para comprovar com maior segurança que os exercícios físicos realizados com pacientes portadores de DRC na diálise trazem benefícios no Kt/V, seria necessário um número superior de participantes. Assim, sugere-se a realização de novos estudos com maior quantidade de pacientes.

\section{Referências}

1. Coelho DM, Castro AM, Tavares HA, Abreu PCB, Glória $\mathrm{RR}$, Duart MH, et al. Efeitos de um programa de exercícios físicos no condicionamento de pacientes em hemodiálise. J Bras Nefrol. 2006:28(3):121-7.

2. Schor N, Srougi M. Nefrologia urologia clínica. São Paulo: Sarvier; 1998.

3. Cruz J, Praxedes JN, Cruz HMM. Nefrologia. São Paulo: Sarvier; 1994.
4. Sociedade Brasileira de Nefrologia. Diretrizes Brasileiras de Doença Renal Crônica. 2004.

5. Peres CPA. Efeito de um programa de exercícios físicos em pacientes em hemodiálise [dissertação]. Londrina: Universidade Estadual de Londrina; 2008.

6. Lima JJG. Doenças cardiovasculares em pacientes renais crônicos tratados por hemodiálise: abordagem prática. São Paulo Med J. 2006;124(1):36-41. PMid:16612461.

7. Barros E. Nefrologia: rotina, diagnóstico e tratamento. 3. ed. Porto Alegre: Artmed; 2006.

8. Daugirdas JT, Blake PG, Ing TS. Handbook of dialysis. 3. ed. Rio de Janeiro: Medsi; 2001.

9. Storer TW, Casaburi R, Sawelson S, Kopple JD. Endurance exercise training during haemodialysis improves strength, power, fatigability and physical performance in maintenance haemodialysis patients. Nephrol Dial Transplant. 2005;20(7):1429-37. doi:10.1093/ndt/gfh784.

10. Riella MC. Princípios de nefrologia e distúrbios hidroeletrolíticos. 4. ed. Rio de Janeiro: Guanabara Koogan S.A; 2003

11. Lopez C, Simmons RL, Mauer SM, Najarian JS, Good RA, Gentry S. Association of renal allograft rejection with vírus infection. Am J Med. 1974:3(56);280-9. doi:10.1016/0002-9343(74)90609-3.

12. Daugirdas JT, Greene T, Depner TA, Leypoldt J, Gotch F, Schulman G, et al. Factors that affect postdialysis rebound in serum urea concentratin, incluing the rate of dialysis: results from the HEMO study. J Am Soc Nephrol. 2004;15:194-203. doi:10.1097/01. ASN.0000103871.20736.0C.

13. Kong CH, Tattersall JE, Greenwood RN, Farrington K. The effect of exercise during haemodialysis on solute removal. Nephrol Dial Transplant. 1999;14:2927-31. doi:10.1093/ndt/14.12.2927.

14. Schneditz D, Van Stone JC, Daugirdas JT. A regional blood circulation alternative to in-series two compartment urea kinetic modeling. ASAIO J. 1993;39(3):M573577. doi:10.1097/00002480-199307000-00086.

15. Schneditz D, Daugirdas JT. Formal analytical solution to a regional blood flow and diffusion based urea kinetic model. ASAIO J. 1994;40(3):M667-73. doi:10.1097/00002480-199407000-00082. 
16. Daugirdas JT, Schneditz D. Overestimation of hemodialysis dose depends on dialysis efficiency by regional blood flow but no by conventional two pool urea kinetic analysis. ASAIO J. 1995;41(3):M719-24.

17. Aires MM. Fisiologia. 2. ed. Rio de Janeiro: Guanabara Koogan S.A; 1999.

18. Kisner C, Colby LA. Exercícios terapêuticos: fundamentos e técnicas. 4. ed. Barueri: Manole; 2005.

19. Leite PF. Fisiologia do exercício, ergometria e condicionamento físico. In: Leite PF. Cardiologia desportiva. 4.ed. São Paulo: Robe Editorial; 2000. p. 37-38.

20. Guyton AC, Hall JE. Tratado de fisiologia médica. 9. ed. Rio de Janeiro: Guanabara Koogan S.A; 1997.

21. Johansen KL. Exercise and chronic kidney disease. Sports Med. 2005;35(6):485-99. doi:10.2165/ 00007256-200535060-00003.

22. Johansen KL. Exercise in the end-stage renal disease population. J Am Soc Nephrol. 2007;18(6):1845-54. doi:10.1681/ASN.2007010009.

23. Marieke CBAV, Mathieu HGG, Roel MH. The effects of a low-to-moderate intensity pre-conditioning exercise programme linked with exercise counselling for sedentary haemodialysis patients in the Netherlands: results of a randomized clinical trial. Nephrol Dial Transplant. 2004;20(1):141-6.

24. Koufaki P, Mercer TH, Naish PF. Effects of exercise training on aerobic an functional capacity of end-stage renal disease patients. Clin Physiol Funct Imaging. 2002;22(2):115-24. doi:10.1046/ j.1365-2281.2002.00405.x.

25. Headley S, Germain M, Mailloux P, Mulhern J, Ashworth $\mathrm{B}$, Burris J, et al. Resistance training improves strength an functional measures in patients with end-stage renal disease. Am J Kidney Dis. 2002;40(2):355-64. doi:10.1053/ajkd.2002.34520.

26. Mercer TH, Crawford C, Gleeson NP, Naish PF. Low-volume exercise rehabilitation improves functional capacity an self-reported functional status of dialysis patients. Am J Phys Med Rehabil. 2002;81(3):162-7. doi:10.1097/00002060-200203000-00002.

27. Oh-Park M, Fast A, Gopal S, Lynn R, Frei G, Drenth R, et al. Exercise for the dialized: aerobic and strength training during hemodialysis. Am J Phys Med Rehabil. 2002; 81(11):814-21.doi:10.1097/00002060-200211000-00003.
28. Storer TW, Casaburi R, Sawelson S, Kopple JD. Endurance exercise training during haemodialysis improves strength, power, fatigability and physical performance in maintenance haemodialysis patients. Nephrol Dial Transplant. 2005;20(7):1429-37. doi:10.1093/ndt/ gfh784.

29. Painter P, Carlson L, Carey S, Paul SM, Myll J. Physical functioning and health-related quality-of-life changes with exercise training in hemodialysis patients. Am J Kidney Dis. 2000;35(3):482-92. doi:10.1016/ S0272-6386(00)70202-2.

30. Kouidi E, Grekas D, Deligiannis A, Tourkantonis A. Outcomes of long-term exercise training in dialysis patients: comparison of two training programs. Clin Nephrol. 2004;61(1)31-8.

31. Carvalho T. Diretriz de reabilitação cardiopulmonar e metabólica: aspectos práticos e de responsabilidades. Arq Bras Cardiol. 2006;86(1):74-82. doi:10.1590/ S0066-782X2006000100011.

32. Deligiannis A. Exercise rehabilitation and skeletal muscle benefits in hemodialysis patients. Clin Nephrol. 2004;61:46-50.

33. Koufaki P, Mercer TH, Naish PF. Effects of exercise training on aerobic an functional capacity of end-stage renal disease patients. Clin Physiol Funct Imaging. 2002;22(2):115-24. doi:10.1046/ j.1365-2281.2002.00405.x.

34. Parsons TL, Toffelmire EB, King-VanVack CE. The effect of an exercise program during hemodialysis on dialysis efficacy, blood pressure and quality of life in end-stage renal disease (ESRD) patients. Clin Neprol. 2004;61(4):261-74.

35. Rizzioli E, Cerretani D, Normanno M, Munaro D, Berto A, Contarello G, et al. Physical exercise during hemodialysis session: effect on quality of life. G Ital Nefrol. 2004;21(30):S236-40. PMid:15750992.

36. Adams GP, Varizi ND. Skeletal muscle disfunction in chronic renal failure: effects of exercise. Am J Physiol Renal. 2006;290:753-61. doi:10.1152/ ajprenal.00296.2005.

37. Banerjee A, Kong $\mathrm{CH}$, Farrington $\mathrm{K}$. The haemodynamic response to submaximal exercise during isovolaemic haemodialysis. Nephrol Dial Transplant. 2004:6(19):3204-6. 
38. Kusumota L, Rodrigues RA, Marques S. Idoso com insuficiência renal crônica: alterações do estado de Saúde. Rev Lat Am Enfermagem. 2004:12(3):525-32. doi:10.1590/S0104-11692004000300011.

39. Martins MRI, Cesarino CB. Atualização sobre programas de educação e reabilitação para pacientes renais crônicos submetidos à hemodiálise. J Bras Nef. 2004;26(1):45-50.

40. Moore GE, Painter PL, Brinker KR, Stray-Gundersen J, Mitchell JH. Cardiovascular response to submaximal stationary cycling during haemodialysis. Am J Kidney Dis. 1998;31(4):631-7. doi:10.1053/ajkd.1998.v31. pm9531179.
41. Moreira PR, Barros EG. Revisão/Atualização em diálise: capacidade e condicionamento físico em pacientes mantidos em hemodiálise. J Bras Nef. 1998;20(2):207-10.

Recebido: 30/03/2012 Received: 03/30/2012

Aprovado: 17/09/2012 Approved: 09/17/2012 